\title{
A Class of Sequences Defined by Weak Ideal Convergence and Musielak-Orlicz Function
}

\author{
Awad A. Bakery ${ }^{1,2}$ \\ ${ }^{1}$ Department of Mathematics, Faculty of Science and Arts, King Abdulaziz University (KAU), \\ P.O. Box 80200, Khulais 21589, Saudi Arabia \\ ${ }^{2}$ Department of Mathematics, Faculty of Science, Ain Shams University, P.O. Box 1156, Abbassia, Cairo 11566, Egypt
}

Correspondence should be addressed to Awad A. Bakery; awad_bakery@yahoo.com

Received 9 October 2013; Accepted 17 March 2014; Published 27 April 2014

Academic Editor: Feyzi Başar

Copyright (c) 2014 Awad A. Bakery. This is an open access article distributed under the Creative Commons Attribution License, which permits unrestricted use, distribution, and reproduction in any medium, provided the original work is properly cited.

We introduced the weak ideal convergence of new sequence spaces combining an infinite matrix of complex numbers and MusielakOrlicz function over normed spaces. We also study some topological properties and inclusion relation between these spaces.

\section{Introduction}

Throughout the paper $\omega, \ell_{\infty}, c, c_{0}$, and $\ell_{p}$ denote the classes of all, bounded, convergent, null, and $p$-absolutely summable sequences of complex numbers. The sets of natural numbers and real numbers will be denoted by $\mathbb{N}, \mathbb{R}$, respectively, and $I$ will denote an admissible ideal in $\mathbb{N} ; X, X^{*}$ will denote a normed linear space $(X,\|\cdot\|)$ and its continuous dual, respectively. Many authors studied various sequence spaces using normed or seminormed linear spaces. In this paper, using an infinite matrix of complex numbers and the notion of weak ideal, we aimed to introduce some new sequence spaces with Musielak-Orlicz function in normed spaces. By an ideal we mean a family $I \subset 2^{Y}$ of subsets of a nonempty set $Y$ satisfying the following: (i) $\phi \in I$; (ii) $A, B \in I$ imply $A \cup B \in I$; (iii) $A \in I, B \subset A$ imply $B \in I$, while an admissible ideal $I$ of $Y$ further satisfies $\{x\} \in I$ for each $x \in Y$. The notion of ideal convergence was introduced first by P. Kostyrko et al. [1] as a generalization of statistical convergence. Given that $I \subset 2^{\mathbb{N}}$ is a nontrivial ideal in $\mathbb{N}$, the sequence $\left(x_{n}\right)_{n \in \mathbb{N}}$ in a normed space $(X ;\|\cdot\|)$ is said to be $I$-convergent to $x \in X$ if, for each $\varepsilon>0$,

$$
A(\varepsilon)=\left\{n \in \mathbb{N}:\left\|x_{n}-x\right\| \geq \varepsilon\right\} \in I .
$$

A sequence $\left(x_{k}\right)$ in a normed space $(X,\|\cdot\|)$ is said to be $I$ bounded if there exists $L>0$ such that

$$
\left\{k \in \mathbb{N}:\left\|x_{k}\right\|>L\right\} \in I \text {. }
$$

A sequence $\left(x_{k}\right)$ in a normed space $(X,\|\cdot\|)$ is said to be $I$ Cauchy if, for each $\varepsilon>0$, there exists a positive integer $m=$ $m(\varepsilon)$ such that

$$
\left\{n \in \mathbb{N}:\left\|x_{n}-x_{m}\right\| \geq \varepsilon\right\} \in I .
$$

Recently different classes of sequences have been introduced using ideal convergence; see [2,3]. Following [4, 5], Pehlivan et al. [6] have introduced the concepts of weak $I$-convergence and weak $I$-Cauchy sequence in a normed space and investigated their basic properties. A sequence $\left(x_{n}\right)_{n \in \mathbb{N}}$ in a normed space $(X ;\|\cdot\|)$ is said to be weak $I$-convergent to $x \in X$ if, for each $\varepsilon>0$ and for each $f \in X^{*}$, the set

$$
A(\varepsilon)=\left\{n \in \mathbb{N}:\left|f\left(x_{n}\right)-f(x)\right| \geq \varepsilon\right\} \in I .
$$

A sequence $\left(x_{n}\right)$ in a normed space $(X,\|\cdot\|)$ is said to be weak $I$-bounded for each $f \in X^{*}$ if there exists $L>0$ such that

$$
\left\{k \in \mathbb{N}:\left|f\left(x_{k}\right)\right|>L\right\} \in I .
$$

A sequence $\left(x_{k}\right)$ in a normed space $(X,\|\cdot\|)$ is said to be weak $I$-Cauchy if, for each $\varepsilon>0$ and for each $f \in X^{*}$, there exists a positive integer $m=m(\varepsilon)$ such that

$$
\left\{k \in \mathbb{N}:\left|f\left(x_{k}\right)-f\left(x_{m}\right)\right| \geq \varepsilon\right\} \in I .
$$

An Orlicz function is a function $M:[0, \infty) \rightarrow[0, \infty)$ which is continuous, nondecreasing, and convex with $M(0)=0$, 
$M(x)>0$ for $x>0$, and $M(x) \rightarrow \infty$, as $x \rightarrow \infty$. If convexity of $M$ is replaced by $M(x+y) \leq M(x)+M(y)$, then it is called a modulus function, introduced by Nakano [7]. Ruckle [8] and Maddox [9] used the idea of a modulus function to construct some spaces of complex sequences. An Orlicz function $M$ is said to satisfy $\Delta_{2}$-condition for all values of $x \geq 0$ if there exists a constant $k>0$, such that $M(2 x) \leq$ $k M(x)$. The $\Delta_{2}$-condition is equivalent to $M(l x) \leq k l M(x)$ for all values of $x$ and for $l>1$. Lindenstrauss and Tzafriri [10] used the idea of an Orlicz function to define the following sequence spaces:

$$
\ell_{M}=\left\{x \in \omega: \sum_{k=1}^{\infty} M\left(\frac{|x(k)|}{\rho}\right)<\infty\right\},
$$

which is a Banach space with the Luxemburg norm defined by

$$
\|x\|=\inf \left\{\rho>0: \sum_{k=1}^{\infty} M\left(\frac{|x(k)|}{\rho}\right) \leq 1\right\} .
$$

The space $\ell_{M}$ is closely related to the space $\ell_{p}$, which is an Orlicz sequence space with $M(x)=x^{p}$ for $1 \leq p<\infty$. Recently different classes of sequences have been introduced using Orlicz functions. See [11-14]. A sequence $\mathscr{M}=\left(M_{k}\right)$ of Orlicz functions $M_{k}$ for all $k \in \mathbb{N}$ is called a Musielak-Orlicz function.

\section{Definitions and Preliminaries}

Let $x=\left(x_{k}\right)$ be a sequence; then $S(x)$ denotes the set of all permutations of the elements of $\left(x_{k}\right)$; that is,

$$
S(x)=\left\{\left(x_{\pi(n)}\right): \pi \text { is a permutation of } \mathbb{N}\right\} .
$$

Definition 1. A sequence space $E$ is said to be symmetric if $S(x) \subset E$ for all $x \in E$.

Definition 2. A sequence space $E$ is said to be normal (or solid) if $\left(\alpha_{k} x_{k}\right) \in E$, whenever $\left(x_{k}\right) \in E$ and for all sequence $\left(\alpha_{k}\right)$ of scalars with $\left|\alpha_{k}\right| \leq 1$ for all $k \in \mathbb{N}$.

Let $K=\left\{k_{1}<k_{2}<\cdots\right\} \subseteq \mathbb{N}$ and let $E$ be a sequence space. A $K$-step space of $E$ is a sequence space $\lambda_{K}^{E}=\left\{\left(x_{k_{n}}\right) \in\right.$ $\left.\omega:\left(k_{n}\right) \in E\right\}$. A canonical preimage of a sequence $x_{k_{n}} \in \lambda_{K}^{E}$ is a sequence $y_{k} \in \omega$ defined as

$$
y_{k}= \begin{cases}x_{k}, & \text { if } k \in K \\ 0, & \text { otherwise }\end{cases}
$$

A canonical preimage of a step space $\lambda_{K}^{E}$ is a set of canonical preimages of all elements in $\lambda_{K}^{E}$; that is, $y$ is in canonical preimage of $\lambda_{K}^{E}$ if and only if $y$ is canonical preimage of some $x \in \lambda_{K}^{E}$.

Definition 3. A sequence space $E$ is said to be monotone if $E$ contains the canonical preimages of all its step spaces.
Lemma 4. Every normal space is monotone.

For any bounded sequence $\left(p_{n}\right)$ of positive numbers, we have the following well known inequality.

If $0 \leq p_{k} \leq \sup _{k} p_{k}=G$ and $D=\max \left(1,2^{G-1}\right)$, then $\left|a_{n}+b_{n}\right|^{p_{n}} \leq D\left(\left|a_{n}\right|^{p_{n}}+\left|b_{n}\right|^{p_{n}}\right)$ for all $k$ and $a_{k}, b_{k} \in \mathbb{C}$.

\section{Main Results}

In this section, we define some new weak ideal convergent sequence spaces and investigate their linear topological structures. We find out some relations related to these sequence spaces. Let $w-I$ be a weak admissible ideal of $\mathbb{N}$, let $\mathscr{M}=$ $\left(M_{j}\right)$ be a Musielak-Orlicz function, and let $X$ and $Y$ be two nonempty subsets of the space $\omega$ of complex sequences. Let $A=\left(a_{k j}\right),(k, j=1,2,3, \ldots)$ be an infinite matrix of complex numbers. We write $A x=\left(A_{j}(x)\right)$ if $A_{j}(x)=\sum_{m=1}^{\infty} a_{j m} x_{m}$ converges for each $j$. Further, let $p=\left(p_{k}\right)$ be any bounded sequence of positive real numbers:

$$
\begin{aligned}
& m[A, \mathscr{M}, p,\|\cdot\|]^{w-I} \\
& =\left\{x \in \omega(X): \forall \varepsilon>0, \forall f \in X^{*},\right. \\
& \left\{k \in \mathbb{N}: \sum_{j=1}^{\infty} a_{k j}\left[M_{j}\left(\left|\frac{f(x)-l}{\rho}\right|\right)\right]^{p_{j}} \geq \varepsilon\right\} \in I \\
& \text { for some } \rho>0, l \in X\} \text {, } \\
& m[A, \mathscr{M}, p,\|\cdot\|]_{0}^{w-I} \\
& =\left\{x \in \omega(X): \forall \varepsilon>0, \forall f \in X^{*},\right. \\
& \left\{k \in \mathbb{N}: \sum_{j=1}^{\infty} a_{k j}\left[M_{j}\left(\left|\frac{f(x)}{\rho}\right|\right)\right]^{p_{j}} \geq \varepsilon\right\} \in I \\
& \text { for some } \rho>0\} \text {, } \\
& \begin{array}{c}
m[A, \mathscr{M}, p,\|\cdot\|]_{\infty} \\
=\{x \in \omega(X):
\end{array} \\
& \exists K>0 \text { s.t. } \sup _{k} \sum_{j=1}^{\infty} a_{k j}\left[M_{j}\left(\left|\frac{f(x)}{\rho}\right|\right)\right]^{p_{j}}<\infty \\
& \text { for some } \rho>0\} \text {, }
\end{aligned}
$$




$$
\begin{aligned}
& m[A, \mathscr{M}, p,\|\cdot\|]_{\infty}^{w-I} \\
& =\{x \in \omega(X): \\
& \exists K>0, \text { s.t. }\{k \in \mathbb{N}: \\
& \left.\sum_{j=1}^{\infty} a_{k j}\left[M_{j}\left(\left|\frac{f(x)}{\rho}\right|\right)\right]^{p_{j}} \geq K\right\} \in I \\
& \text { for some } \left.\rho>0 \text { and each } f \in X^{*}\right\} .
\end{aligned}
$$

Let us consider a few special cases of the above sets.

(1) If $M_{k}(x)=M(x)$ for all $k \in \mathbb{N}$, then the above classes of sequences are denoted by $m[A, M, p$, $\|\cdot\|]^{w-I}, m[A, M, p,\|\cdot\|]_{0}^{w-I}, m[A, M, p,\|\cdot\|]_{\infty}$, and $m[A, M, p,\|\cdot\|]_{\infty}^{w-I}$, respectively.

(2) If $p_{k}=1$ for all $k \in \mathbb{N}$, then the above classes of sequences are denoted by $m[A, \mathscr{M},\|\cdot\|]^{w-I}$, $m[A, \mathscr{M},\|\cdot\|]_{0}^{w-I}, m[A, \mathscr{M},\|\cdot\|]_{\infty}$, and $m[A, \mathscr{M}$, $\|\cdot\|]_{\infty}^{w-I}$, respectively.

(3) If $M_{k}(x)=x$ for all $k \in \mathbb{N}$ and $x \in[0, \infty[$, then the above classes of sequences are denoted by $m[A, p$, $\|\cdot\|]^{w-I}, m[A, p,\|\cdot\|]_{0}^{w-I}, m[A, p,\|\cdot\|]_{\infty}$, and $m[A, p$, $\|\cdot\|]_{\infty}^{w-I}$, respectively.

(4) If we take $M_{k}(x)=M(x)$ for all $k \in \mathbb{N}$ and $A=\left(a_{k j}\right)$ as

$$
a_{k j}= \begin{cases}\frac{1}{k}, & k \geq j \\ 0, & \text { otherwise }\end{cases}
$$

then we denote the above classes of sequences by $m[C, M, p,\|\cdot\|]^{w-I}, m[C, M, p,\|\cdot\|]_{0}^{w-I}, m[C, M, p, \| \cdot$ $\|]_{\infty}$, and $m[C, M, p,\|\cdot\|]_{\infty}^{w-I}$, respectively.

(5) If we take $M_{k}(x)=M(x)$ and $A=\left(a_{k j}\right)$ as

$$
a_{k j}= \begin{cases}\frac{1}{\lambda_{k}}, & j \in I_{k}=\left[k-\lambda_{k}+1, k\right] \\ 0, & \text { otherwise, }\end{cases}
$$

where $\left(\lambda_{k}\right)$ is a nondecreasing sequence of positive numbers tending to $\infty, \lambda_{1}=1$, and $\lambda_{k+1} \leq \lambda_{k}+1$, then we denote the above classes of sequences by $m[\lambda, M, p,\|\cdot\|]^{w-I}, m[\lambda, M, p,\|\cdot\|]_{0}^{w-I}, m[\lambda, M, p$, $\|\cdot\|]_{\infty}$, and $m[\lambda, M, p,\|\cdot\|]_{\infty}^{w-I}$.

(6) By a lacunary $\theta=\left(j_{r}\right), r=0,1,2, \ldots$, where $j_{0}=0$, we will mean an increasing sequence of nonnegative integers with $j_{r}-j_{r-1} \rightarrow \infty$ as $r \rightarrow \infty$. The interval determined by $\theta$ will be denoted by $\left.I_{r}=\right] j_{r-1}, j_{r}$ ] and $h_{r}=j_{r}-j_{r-1}$ and let $A=\left(a_{k j}\right)$ as

$$
a_{k j}= \begin{cases}\frac{1}{h_{r}}, & \left.\left.j \in I_{r}=\right] j_{r-1}, j_{r}\right] \\ 0, & \text { otherwise. }\end{cases}
$$

Then we denote the above classes of sequences by $m[\theta, M, p,\|\cdot\|]^{w-I}, m[\theta, M, p,\|\cdot\|]_{0}^{w-I}, m[\theta, M, p$, $\|\cdot\|]_{\infty}$, and $m[\theta, M, p,\|\cdot\|]_{\infty}^{w-I}$, respectively.

(7) If $M_{k}(x)=M(x)$, for all $k \in \mathbb{N}$ and $A=I$, then the above classes of sequences are denoted by $m[M, p$, $\|\cdot\|]^{w-I}, m[M, p,\|\cdot\|]_{0}^{w-I}, m[M, p,\|\cdot\|]_{\infty}$, and $m[M, p,\|\cdot\|]_{\infty}^{w-I}$, respectively.

Theorem 5. The spaces $m[A, \mathscr{M}, p,\|\cdot\|]^{w-I}, m[A, \mathscr{M}, p$, $\|\cdot\|]_{0}^{w-I}$, and $m[A, \mathscr{M}, p,\|\cdot\|]_{\infty}^{w-I}$ are linear spaces.

Proof. We will prove the assertion for $m[A, \mathscr{M}, p,\|\cdot\|]_{0}^{w-I}$; the others can be proved similarly. Assume that $x=\left(x_{k}\right), y=$ $\left(y_{k}\right) \in m[A, \mathscr{M}, p,\|\cdot\|]_{0}^{w-I}$, and $\alpha, \beta \in \mathbb{C}$. Then, there exist $\rho_{1}$ and $\rho_{2}$ such that the sets

$$
\begin{gathered}
\left\{k \in \mathbb{N}: \sum_{j=1}^{\infty} a_{k j}\left[M_{j}\left(\left|\frac{f(x)}{\rho_{1}}\right|\right)\right]^{p_{j}} \geq \frac{\varepsilon}{2}\right\} \in I, \\
\left\{k \in \mathbb{N}: \sum_{j=1}^{\infty} a_{k j}\left[M_{j}\left(\left|\frac{f(y)}{\rho_{2}}\right|\right)\right]^{p_{j}} \geq \frac{\varepsilon}{2}\right\} \in I .
\end{gathered}
$$

Since $f$ is linear and the Orlicz function $M_{j}$ is convex for all $j \in \mathbb{N}$, the following inequality holds:

$$
\begin{aligned}
\sum_{j=1}^{\infty} a_{k j} & {\left[M_{j}\left(\left|\frac{f(\alpha x+\beta y)}{|\alpha| \rho_{1}+|\beta| \rho_{2}}\right|\right)\right]^{p_{j}} } \\
\leq & D \sum_{j=1}^{\infty} a_{k j} \frac{|\alpha| \rho_{1}}{|\alpha| \rho_{1}+|\beta| \rho_{2}}\left[M_{j}\left(\left|\frac{f(x)}{\rho_{1}}\right|\right)\right]^{p_{j}} \\
& +D \sum_{j=1}^{\infty} a_{k j} \frac{|\beta| \rho_{2}}{|\alpha| \rho_{1}+|\beta| \rho_{2}}\left[M_{j}\left(\left|\frac{f(y)}{\rho_{2}}\right|\right)\right]^{p_{j}} \\
\leq & D L \sum_{j=1}^{\infty} a_{k j}\left[M_{j}\left(\left|\frac{f(x)}{\rho_{1}}\right|\right)\right]^{p_{j}} \\
& +D L \sum_{j=1}^{\infty} a_{k j}\left[M_{j}\left(\left|\frac{f(y)}{\rho_{2}}\right|\right)\right]^{p_{j}}
\end{aligned}
$$


where $L=\max \left\{|\alpha| \rho_{1} /\left(|\alpha| \rho_{1}+|\beta| \rho_{2}\right),|\beta| \rho_{2} /\left(|\alpha| \rho_{1}+|\beta| \rho_{2}\right)\right\}$. On the other hand from the above inequality we get

$$
\begin{gathered}
\left\{k \in \mathbb{N}: \sum_{j=1}^{\infty} a_{k j}\left[M_{j}\left(\left|\frac{f(\alpha x+\beta y)}{|\alpha| \rho_{1}+|\beta| \rho_{2}}\right|\right)\right]^{p_{j}} \geq \varepsilon\right\} \\
\subseteq\left\{k \in \mathbb{N}: D L \sum_{j=1}^{\infty} a_{k j}\left[M_{j}\left(\left|\frac{f(x)}{\rho_{1}}\right|\right)\right]^{p_{j}} \geq \frac{\varepsilon}{2}\right\} \\
\cup\left\{k \in \mathbb{N}: D L \sum_{j=1}^{\infty} a_{k j}\left[M_{j}\left(\left|\frac{f(y)}{\rho_{2}}\right|\right)\right]^{p_{j}} \geq \frac{\varepsilon}{2}\right\} .
\end{gathered}
$$

Since the two sets on the right hand side belong to $I$, this completes the proof.

Theorem 6. The spaces $m[A, \mathscr{M}, p,\|\cdot\|]^{w-I}, m[A, \mathscr{M}, p$, $\|\cdot\|]_{0}^{w-I}$, and $m[A, \mathscr{M}, p,\|\cdot\|]_{\infty}^{w-I}$ are paranormed spaces with respect to the paranorm $g$ defined by

$$
g(x)=\inf _{k}\left\{\rho^{p_{k} / H}:\left[\sum_{j=1}^{\infty} a_{k j}\left[M_{j}\left(\left|\frac{f(x)}{\rho}\right|\right)\right]^{p_{j}}\right]^{1 / H}\right.
$$$$
\leq 1 \text { for some } \rho>0\} \text {, }
$$

where $H=\max \left\{1, \sup _{k} p_{k}\right\}$.

Proof. Clearly $g(-x)=g(x)$ and $g(x)=0 \Leftrightarrow x=\Theta$, where $\Theta$ is the zero element of $X$. Let $x=\left(x_{k}\right)$ and $y=\left(y_{k}\right) \epsilon$ $m[A, \mathscr{M}, p,\|\cdot\|]_{0}^{w-I}$. Then, for $\rho>0$, we set

$$
\begin{aligned}
& A_{1}=\left\{\rho: \sum_{j=1}^{\infty} a_{k j}\left[M_{j}\left(\left|\frac{f(x)}{\rho}\right|\right)\right]^{p_{j}} \leq 1\right\}, \\
& A_{2}=\left\{\rho: \sum_{j=1}^{\infty} a_{k j}\left[M_{j}\left(\left|\frac{f(y)}{\rho}\right|\right)\right]^{p_{j}} \leq 1\right\} .
\end{aligned}
$$

Let $\rho_{1} \in A_{1}, \rho_{2} \in A_{2}$, and $\rho=\rho_{1}+\rho_{2}$; then we have

$$
\begin{aligned}
\sum_{j=1}^{\infty} a_{k j} & {\left[M_{j}\left(\left|\frac{f(x+y)}{\rho}\right|\right)\right]^{p_{j}} } \\
\leq & \frac{\rho_{1}}{\rho_{1}+\rho_{2}} \sum_{j=1}^{\infty} a_{k j}\left[M_{j}\left(\left|\frac{f(x)}{\rho_{1}}\right|\right)\right]^{p_{j}} \\
& +\frac{\rho_{2}}{\rho_{1}+\rho_{2}} \sum_{j=1}^{\infty} a_{k j}\left[M_{j}\left(\left|\frac{f(y)}{\rho_{2}}\right|\right)\right]^{p_{j}} \leq 1,
\end{aligned}
$$

$$
\begin{aligned}
g(x+y)= & \inf \left\{\left(\rho_{1}+\rho_{2}\right)^{p_{k} / H}: \rho_{1} \in A_{1}, \rho_{2} \in A_{2}\right\} \\
\leq & \inf \left\{\left(\rho_{1}\right)^{p_{k} / H}: \rho_{1} \in A_{1}\right\} \\
& +\inf \left\{\left(\rho_{2}\right)^{p_{k} / H}: \rho_{2} \in A_{2}\right\} \\
= & g(x)+g(y) .
\end{aligned}
$$

Let $\lambda^{t} \rightarrow \lambda$ where $\lambda^{t}, \lambda \in \mathbb{C}$, and let $g\left(x^{t}-x\right) \rightarrow 0$ as $t \rightarrow \infty$. We have to show that $g\left(\lambda^{t} x^{t}-\lambda x\right) \rightarrow 0$ as $t \rightarrow \infty$. We set

$$
\begin{aligned}
& A_{3}=\left\{\rho_{t}: \sum_{j=1}^{\infty} a_{k j}\left[M_{j}\left(\left|\frac{f(x)}{\rho_{t}}\right|\right)\right]^{p_{j}} \leq 1\right\}, \\
& A_{4}=\left\{\rho_{t}^{1}: \sum_{j=1}^{\infty} a_{k j}\left[M_{j}\left(\left|\frac{f(y)}{\rho_{t}^{1}}\right|\right)\right]^{p_{j}} \leq 1\right\} .
\end{aligned}
$$

If $\rho_{t} \in A_{3}$ and $\rho_{t}^{1} \in A_{4}$, by using nondecreasing and convexity of the Orlicz function $M_{j}$ for all $j \in \mathbb{N}$, we obtain that

$$
\begin{gathered}
\sum_{j=1}^{\infty} a_{k j}\left[M_{j}\left(\left|\frac{f\left(\lambda^{t} x^{t}-\lambda x\right)}{\left|\lambda^{t}-\lambda\right| \rho_{t}+|\lambda| \rho_{t}^{1}}\right|\right)\right]^{p_{j}} \\
\leq \sum_{j=1}^{\infty} a_{k j}\left[M _ { j } \left(\left|\frac{f\left(\lambda^{t} x^{t}-\lambda x^{t}\right)}{\left|\lambda^{t}-\lambda\right| \rho_{t}+|\lambda| \rho_{t}^{1}}\right|\right.\right. \\
\left.\left.\quad+\left|\frac{f\left(\lambda x^{t}-\lambda x\right)}{\left|\lambda^{t}-\lambda\right| \rho_{t}+|\lambda| \rho_{t}^{1}}\right|\right)\right]^{p_{j}} \\
\leq \frac{\left|\lambda^{t}-\lambda\right| \rho_{t}}{\left|\lambda^{t}-\lambda\right| \rho_{t}+|\lambda| \rho_{t}^{1}} \sum_{j=1}^{\infty} a_{k j}\left[M_{j}\left(\left|\frac{f\left(x^{t}\right)}{\rho_{t}}\right|\right)\right]^{p_{j}} \\
+\frac{|\lambda| \rho_{t}^{1}}{\left|\lambda^{t}-\lambda\right| \rho_{t}+|\lambda| \rho_{t}^{1}} \sum_{j=1}^{\infty} a_{k j}\left[M_{j}\left(\left|\frac{f\left(x^{t}-x\right)}{\rho_{t}^{1}}\right|\right)\right]^{p_{j}} .
\end{gathered}
$$

From the above inequality, it follows that

$$
\sum_{j=1}^{\infty} a_{k j}\left[M_{j}\left(\left|\frac{f\left(\lambda^{t} x^{t}-\lambda x\right)}{\left|\lambda^{t}-\lambda\right| \rho_{t}+|\lambda| \rho_{t}^{1}}\right|\right)\right]^{p_{j}} \leq 1,
$$

and consequently

$$
\begin{aligned}
& g\left(\lambda^{t} x^{t}-\lambda x\right) \\
& \quad=\inf \left\{\left(\left|\lambda^{t}-\lambda\right| \rho_{t}+|\lambda| \rho_{t}^{1}\right)^{p_{k} / H}: \rho_{t} \in A_{3}, \rho_{t}^{1} \in A_{4}\right\}
\end{aligned}
$$




$$
\begin{aligned}
\leq & \left|\lambda^{t}-\lambda\right|^{p_{k} / H} \inf \left\{\left(\rho_{t}\right)^{p_{k} / H}: \rho_{t} \in A_{3}\right\} \\
& +|\lambda|^{p_{k} / H} \inf \left\{\left(\rho_{t}^{1}\right)^{p_{k} / H}: \rho_{t}^{1} \in A_{4}\right\} \\
\leq & \max \left\{\left|\lambda^{t}-\lambda\right|,\left|\lambda^{t}-\lambda\right|^{p_{k} / H}\right\} g\left(x^{t}\right) \\
& +\max \left\{|\lambda|,|\lambda|^{p_{k} / H}\right\} g\left(x^{t}-x\right) .
\end{aligned}
$$

Note that $g\left(x^{t}\right) \leq g(x)+g\left(x^{t}-x\right)$ for all $t \in \mathbb{N}$. Hence, by our assumption, the right hand of (24) tends to 0 as $t \rightarrow \infty$, and the result follows. This completes the proof of the theorem.

Theorem 7. Let $\mathscr{M}=\left(M_{j}\right), \mathscr{M}^{\prime}=\left(M_{j}^{\prime}\right)$, and $\mathscr{M}^{\prime \prime}=\left(M_{j}^{\prime \prime}\right)$ be Musielak-Orlicz functions. Then, the following hold:

(a) $m\left[A, \mathscr{M}^{\prime}, p,\|\cdot\|\right]_{0}^{w-I} \subseteq m\left[A, \mathscr{M} \cdot \mathscr{M}^{\prime}, p,\|\cdot\|\right]_{0}^{w-I}$, provided $p=\left(p_{k}\right)$ be such that $G_{0}=\inf p_{k}>0$,

(b) $m\left[A, \mathscr{M}^{\prime}, p,\|\cdot\|\right]_{0}^{w-I} \subseteq m\left[A, \mathscr{M}^{\prime}+\mathscr{M}^{\prime \prime}, p,\|\cdot\|\right]_{0}^{w-I}$.

Proof. (a) Let $\varepsilon>0$ be given. Choose $\varepsilon_{1}>0$ such that $\sup _{k}\left(\sum_{j=1}^{\infty} a_{k j}\right) \max \left\{\varepsilon_{1}^{G}, \varepsilon_{1}^{G^{0}}\right\}<\varepsilon$. Using the continuity of the Orlicz function $M$, choose $0<\delta<1$ such that $0<t<\delta$ implies that $M(t)<\varepsilon_{1}$.

Let $x=\left(x_{k}\right)$ be any element in $m\left[A, \mathscr{M}^{\prime}, p,\|\cdot\|\right]_{0}^{w-I}$; put

$$
A_{\delta}=\left\{k \in \mathbb{N}: \sum_{j=1}^{\infty} a_{k j}\left[M_{j}^{\prime}\left(\left|\frac{f(x)}{\rho_{1}}\right|\right)\right]^{p_{j}} \geq \delta^{G}\right\} \text {. }
$$

Then, by definition of ideal convergent, we have the set $A_{\delta} \in$ $I$. If $n \notin A_{\delta}$, then we have

$$
\begin{aligned}
\sum_{j=1}^{\infty} a_{k j}\left[M_{j}^{\prime}\left(\left|\frac{f(x)}{\rho_{1}}\right|\right)\right]^{p_{j}}<\delta^{G} & \Longrightarrow\left[M_{j}^{\prime}\left(\left|\frac{f(x)}{\rho_{1}}\right|\right)\right]^{p_{j}}<\delta^{G} \\
& \Longrightarrow M_{j}^{\prime}\left(\left\|\frac{f(x)}{\rho_{1}}\right\|\right)<\delta .
\end{aligned}
$$

Using the continuity of the Orlicz function $M_{j}$ for all $j$ and the relation (26), we have

$$
M_{j}\left[M_{j}^{\prime}\left(\left|\frac{f(x)}{\rho_{1}}\right|\right)\right]<\varepsilon_{1} .
$$

Consequently, we get

$$
\begin{gathered}
\sum_{j=1}^{\infty} a_{k j}\left[M_{j}^{\prime}\left(\left|\frac{f(x)}{\rho_{1}}\right|\right)\right]^{p_{j}}<\sup _{k}\left(\sum_{j=1}^{\infty} a_{k j}\right) \max \left\{\varepsilon_{1}^{G}, \varepsilon_{1}^{G_{0}}\right\}<\varepsilon \\
\Longrightarrow \sum_{j=1}^{\infty} a_{k j}\left[M_{j} M_{j}^{\prime}\left(\left|\frac{f(x)}{\rho_{1}}\right|\right)\right]^{p_{j}}<\varepsilon .
\end{gathered}
$$

This shows that

$$
\left\{k \in \mathbb{N}: \sum_{j=1}^{\infty} a_{k j}\left[M_{j} M_{j}^{\prime}\left(\left|\frac{f(x)}{\rho_{1}}\right|\right)\right]^{p_{j}} \geq \varepsilon\right\} \subseteq A_{\delta} \in I .
$$

This proves the assertion.

(b) Let $x=\left(x_{k}\right)$ be any element in $m\left[A, \mathscr{M}^{\prime}, p,\|\cdot\|\right]_{0}^{w-I}$. Then, by the following inequality, the results follow:

$$
\begin{gathered}
\sum_{j=1}^{\infty} a_{k j}\left[\left(M_{j}^{\prime}+M_{j}^{\prime \prime}\right)\left(\left|\frac{f(x)}{\rho_{1}}\right|\right)\right]^{p_{j}} \\
\leq D \sum_{j=1}^{\infty} a_{k j}\left[M_{j}^{\prime}\left(\left|\frac{f(x)}{\rho_{1}}\right|\right)\right]^{p_{j}} \\
+D \sum_{j=1}^{\infty} a_{k j}\left[M_{j}^{\prime \prime}\left(\left|\frac{f(x)}{\rho_{1}}\right|\right)\right]^{p_{j}} .
\end{gathered}
$$

Theorem 8. Let $0<p_{k} \leq q_{k}$ for all $k \in \mathbb{N}$; then $m[A, \mathscr{M}, p$, $\|\cdot\|]_{\infty} \subseteq m[A, \mathscr{M}, q,\|\cdot\|]_{\infty}$.

Proof. Let $x=\left(x_{j}\right) \in m[A, \mathscr{M}, p,\|\cdot\|]_{\infty}$; then there exists some $\rho>0$ such that

$$
\sup _{k} \sum_{j=1}^{\infty} a_{k j}\left[M_{j}\left(\left|\frac{f(x)}{\rho}\right|\right)\right]^{p_{j}}<\infty .
$$

This implies that

$$
M_{j}\left(\left|\frac{f(x)}{\rho}\right|\right)<1
$$

for sufficiently large value of $j$. Since $M_{j}$ for all $j \in \mathbb{N}$ is nondecreasing, we get

$$
\begin{aligned}
\sup _{k} \sum_{j=1}^{\infty} a_{k j}\left[M_{j}\left(\left|\frac{f(x)}{\rho}\right|\right)\right]^{q_{j}} \\
\quad \leq \sup _{k} \sum_{j=1}^{\infty} a_{k j}\left[M_{j}\left(\left|\frac{f(x)}{\rho}\right|\right)\right]^{p_{j}}<\infty .
\end{aligned}
$$

Thus, $x \in m[A, \mathscr{M}, q,\|\cdot\|]_{\infty}$. This completes the proof of the theorem.

Theorem 9. (i) If $0<\inf p_{k} \leq p_{k}<1$, then $m$ [A, $M, p$, $\|\cdot\|]_{\infty} \subseteq m[A, \mathscr{M},\|\cdot\|]_{\infty}$.

(ii) If $0<p_{k} \leq \sup _{k} p_{k}<\infty$, then $m[A, \mathscr{M} \text {, }\|\cdot\|]_{\infty} \subseteq$ $m[A, \mathscr{M}, p,\|\cdot\|]_{\infty}$.

Proof. (i) Let $x=\left(x_{j}\right) \in m[A, \mathscr{M}, p,\|\cdot\|]_{\infty}$; since $0<$ $\inf _{k} p_{k} \leq p_{k}<1$, then we have

$$
\sup _{k} \sum_{j=1}^{\infty} a_{k j} M_{j}\left(\left|\frac{f(x)}{\rho}\right|\right) \leq \sup _{k} \sum_{j=1}^{\infty} a_{k j}\left[M_{j}\left(\left|\frac{f(x)}{\rho}\right|\right)\right]^{p_{j}}<\infty,
$$

and hence $x \in m[A, \mathscr{M},\|\cdot\|]_{\infty}$. 
(ii) Let $0<p_{k} \leq \sup _{k} p_{k}<\infty$ and $x=\left(x_{j}\right) \in m[A, \mathscr{M}$, $\|\cdot\|]_{\infty}$. Then for each $0<\varepsilon<1$ there exists a positive integer $j_{0}$ such that

$$
\sup _{k} \sum_{j=1}^{\infty} a_{k j} M_{j}\left(\left|\frac{f(x)}{\rho}\right|\right) \leq \varepsilon<1
$$

for all $j \geq j_{0}$. This implies that

$$
\sup _{k} \sum_{j=1}^{\infty} a_{k j}\left[M_{j}\left(\left|\frac{f(x)}{\rho}\right|\right)\right]^{p_{j}} \leq \sup _{k} \sum_{j=1}^{\infty} a_{k j} M_{j}\left(\left|\frac{f(x)}{\rho}\right|\right)<\infty .
$$

Thus $x \in m[A, \mathscr{M}, p,\|\cdot\|]_{\infty}$ and this completes the proof.

Theorem 10. For any sequence of Orlicz functions $\mathscr{M}=\left(M_{j}\right)$ which satisfies $\Delta_{2}$-condition, one has $m[A, p,\|\cdot\|]^{w-I} \subset$ $m[A, \mathscr{M}, p,\|\cdot\|]^{w-I}$.

Proof. Let $x=\left(x_{j}\right) \in m[A, p,\|\cdot\|]^{w-I}$, and let $\varepsilon>0$ be given. Then, there exists $\rho>0$ such that the set

$$
\left\{k \in \mathbb{N}: \sum_{j=1}^{\infty} a_{k j}\left[M_{j}\left(\left|\frac{f(x)-l}{\rho}\right|\right)\right]^{p_{j}} \geq \varepsilon\right\} \in I
$$

for some $l$.

By taking $y_{j}=|(f(x)-l) / \rho|$ and let $\varepsilon>0$ and choose $\delta$ with $0<\delta<1$ such that $M_{j}(t)<\varepsilon$ for all $j \in \mathbb{N}$ and for $0 \leq t \leq \delta$. Consider

$$
\sum_{j=1}^{\infty}\left[M_{j}\left(y_{j}\right)\right]^{p_{j}}=\sum_{j=1, y_{j} \leq \delta}^{\infty}\left[M_{j}\left(y_{j}\right)\right]^{p_{j}}+\sum_{j=1, y_{j}>\delta}^{\infty}\left[M_{j}\left(y_{j}\right)\right]^{p_{j}} .
$$

Since $M_{j}$ is continuous for all $n \in \mathbb{N}$, we have

$$
\sum_{j \in I_{k}, y_{j} \leq \delta}\left[M_{j}\left(y_{j}\right)\right]^{p_{j}}<\varepsilon .
$$

For $y_{j}>\delta$, we use the fact that $y_{j}<\left(y_{j} / \delta\right)<1+\left(y_{j} / \delta\right)$. Since $\mathscr{M}=\left(M_{j}\right)$ is nondecreasing and convex, it follows that

$$
M_{j}\left(y_{j}\right)<M_{j}\left(1+\frac{y_{j}}{\delta}\right)<\frac{1}{2} M_{j}(2)+\frac{1}{2} M_{j}\left(\frac{2 y_{j}}{\delta}\right) .
$$

Since $\mathscr{M}=\left(M_{j}\right)$ satisfies $\Delta_{2}$-condition,

$$
M_{j}\left(y_{j}\right)<\frac{y_{j}}{2 \delta} L M_{j}(2)+\frac{y_{j}}{2 \delta} L M_{j}(2)=\frac{y_{j}}{\delta} L M_{j}(2) .
$$

Hence

$$
\begin{aligned}
& \sum_{j=1, y_{j}>\delta}^{\infty}\left[M_{j}\left(y_{j}\right)\right]^{p_{j}} \\
& \quad<\max \left\{1, \sup _{j}\left(L \delta^{-1} M_{j}(2)\right)^{p_{j}}\right\} \sum_{j=1, y_{j}>\delta}^{\infty}\left(y_{j}\right)^{p_{j}} .
\end{aligned}
$$

By putting (39) and (42) in (38), we get

$$
\begin{aligned}
& \sum_{j=1}^{\infty}\left[M_{j}\left(y_{j}\right)\right]^{p_{j}} \\
& \quad<\varepsilon+\max \left\{1, \sup _{j}\left(L \delta^{-1} M_{j}(2)\right)^{p_{j}}\right\} \sum_{j=1, y_{j}>\delta}^{\infty}\left(y_{j}\right)^{p_{j}} .
\end{aligned}
$$

This proves that $m[A, p,\|\cdot\|]^{w-I} \subset m[A, \mathscr{M}, p,\|\cdot\|]^{w-I}$.

Theorem 11. Let $0<p_{n} \leq q_{n}<1$ and let $\left(q_{n} / p_{n}\right)$ be bounded; then

$$
m[A, \mathscr{M}, q,\|\cdot\|]^{w-I} \subseteq m[A, \mathscr{M}, p,\|\cdot\|]^{w-I} .
$$

Proof. Let $x=\left(x_{j}\right) \in m[A, \mathscr{M}, q,\|\cdot\|]_{\infty}$; we put $y_{j}=$ $\left[M_{j}(|(f(x)-l) / \rho|)\right]^{q_{j}}$ and $\beta_{j}=p_{j} / q_{j}$ for all $j \in \mathbb{N}$. Then $0<\beta_{j} \leq 1$ for all $j \in \mathbb{N}$. Let $\beta$ be such that $0<\beta \leq \beta_{j}$ for all $j \in \mathbb{N}$. Define the sequences $\left(a_{j}\right)$ and $\left(b_{j}\right)$ as follows: for $y_{j} \geq 1$, let $a_{j}=y_{j}$ and $b_{j}=0$; for $y_{j}<1$ let $a_{j}=0$ and $b_{j}=y_{j}$. Then clearly, for all $j \in \mathbb{N}$ we have $y_{j}=a_{j}+b_{j}, y_{j}^{\beta_{j}}=a_{j}^{\beta_{j}}+b_{j}^{\beta_{j}}$, $a_{j}^{\beta_{j}} \leq a_{j} \leq y_{j}$, and $b_{j}^{\beta_{j}} \leq b_{j}^{\beta}$. Therefore, we have

$$
\sum_{j=1}^{\infty} a_{k j} y_{j}^{\beta_{j}} \leq \sum_{j=1}^{\infty} a_{k j} y_{j} \leq\left[\sum_{j=1}^{\infty} a_{k j} y_{j}\right]^{\beta}
$$

Hence $x \in m[A, \mathscr{M}, p,\|\cdot\|]_{\infty}$.

Theorem 12. For any two sequences $p=\left(p_{k}\right)$ and $q=\left(q_{k}\right)$ of positive real numbers and for any two norms $\|\cdot\|_{1}$ and $\|\cdot\|_{2}$ on $X$, the following holds:

$$
Z\left[A, \mathscr{M}, p,\|\cdot\|_{1}\right] \cap Z\left[A, \mathscr{M}, q,\|\cdot\|_{2}\right] \neq \phi,
$$

where $Z=m^{w-I}, m_{0}^{w-I}, m_{\infty}^{w-I}$, and $m_{\infty}$.

Proof. Proof of the theorem is obvious, because the zero element belongs to each of the sequence spaces involved in the intersection.

Theorem 13. The sequence spaces $Z[A, \mathscr{M}, p,\|\cdot\|]$ are normal as well as monotone, where $Z=m_{0}^{w-I}, m_{\infty}^{w-I}$.

Proof. We will give the proof for $m[A, \mathscr{M}, p,\|\cdot\|]_{0}^{w-I}$ only. Let $x=\left(x_{j}\right) \in m[A, \mathscr{M}, p,\|\cdot\|]_{0}^{w-I}$ and let $\alpha=\left(\alpha_{j}\right)$ be a sequence of scalars such that $\left|\alpha_{j}\right| \leq 1$ for all $j \in \mathbb{N}$. Then, we have

$$
\begin{aligned}
& \left\{k \in \mathbb{N}: \sum_{j=1}^{\infty} a_{k j}\left[M_{j}\left(\left|\frac{f(\alpha x)}{\rho}\right|\right)\right]^{p_{j}}\right\} \\
& \subseteq\left\{k \in \mathbb{N}: E \sum_{j=1}^{\infty} a_{k j}\left[M_{j}\left(\left|\frac{f(x)}{\rho}\right|\right)\right]^{p_{j}}\right\},
\end{aligned}
$$

where $E=\max \left\{1,\left|\alpha_{j}\right|^{G_{0}}\right\}$; hence, $\alpha x=\left(\alpha_{j} x_{j}\right) \in$ $m[A, \mathscr{M}, p,\|\cdot\|]_{0}^{w-I}$. By Lemma 4 , we have that the space $m[A, \mathscr{M}, p,\|\cdot\|]_{0}^{w-I}$ is monotone. 
Note. It is clear from the definitions that

$$
\begin{aligned}
m[A, \mathscr{M}, p,\|\cdot\|]_{0}^{w-I} & \subseteq m[A, \mathscr{M}, p,\|\cdot\|]^{w-I} \\
& \subseteq m[A, \mathscr{M}, p,\|\cdot\|]_{\infty}^{w-I} .
\end{aligned}
$$

\section{Conflict of Interests}

The author declares that there is no conflict of interests regarding the publication of this paper.

\section{Acknowledgments}

Many thanks are due to the editor and anonymous referee for careful reading of the paper and valuable suggestions which helped in improving an earlier version of it.

\section{References}

[1] P. Kostyrko, T. Šalát, and W. Wilczyński, "On I-convergence," Real Analysis Exchange, vol. 26, no. 2, pp. 669-685, 2000-2001.

[2] A. Şahiner, M. Gürdal, S. Saltan, and H. Gunawan, "Ideal convergence in 2-normed spaces," Taiwanese Journal of Mathematics, vol. 11, no. 5, pp. 1477-1484, 2007.

[3] A. Şahiner, M. Gürdal, and T. Yigit, "Ideal convergence characterization of the completion of linear $n$-normed spaces," Computers \& Mathematics with Applications, vol. 61, no. 3, pp. 683-689, 2011.

[4] V. K. Bhardwaj and I. Bala, "On weak statistical convergence," International Journal of Mathematics and Mathematical Sciences, vol. 2007, Article ID 38530, 9 pages, 2007.

[5] J. Connor, M. Ganichev, and V. Kadets, "A characterization of Banach spaces with separable duals via weak statistical convergence," Journal of Mathematical Analysis and Applications, vol. 244, no. 1, pp. 251-261, 2000.

[6] S. Pehlivan, C. Şençimen, and Z. H. Yaman, "On weak ideal convergence in normed spaces," Journal of Interdisciplinary Mathematics, vol. 13, no. 2, pp. 153-162, 2010.

[7] H. Nakano, "Concave modulars," Journal of the Mathematical Society of Japan, vol. 5, pp. 29-49, 1953.

[8] W. H. Ruckle, "FK spaces in which the sequence of coordinate vectors is bounded," Canadian Journal of Mathematics, vol. 25, pp. 973-978, 1973.

[9] I. J. Maddox, "Sequence spaces defined by a modulus," Mathematical Proceedings of the Cambridge Philosophical Society, vol. 100, no. 1, pp. 161-166, 1986.

[10] J. Lindenstrauss and L. Tzafriri, "On Orlicz sequence spaces," Israel Journal of Mathematics, vol. 10, pp. 379-390, 1971.

[11] M. Güngör and M. Et, " $\Delta^{r}$-strongly almost summable sequences defined by Orlicz functions," Indian Journal of Pure and Applied Mathematics, vol. 34, no. 8, pp. 1141-1151, 2003.

[12] M. Et, Y. Altin, B. Choudhary, and B. C. Tripathy, "On some classes of sequences defined by sequences of Orlicz functions," Mathematical Inequalities \& Applications, vol. 9, no. 2, pp. 335342, 2006.

[13] M. Gürdal and A. Şahiner, "New sequence spaces in $n$-normed spaces with respect to an Orlicz function," The Aligarh Bulletin of Mathematics, vol. 27, no. 1, pp. 53-58, 2008.
[14] B. C. Tripathy, Y. Altin, and M. Et, "Generalized difference sequence spaces on seminormed space defined by Orlicz functions," Mathematica Slovaca, vol. 58, no. 3, pp. 315-324, 2008. 


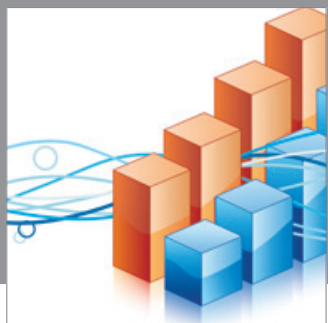

Advances in

Operations Research

mansans

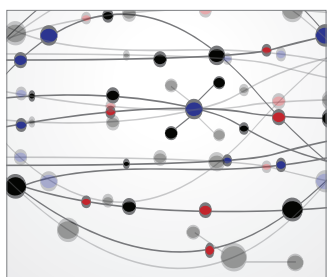

The Scientific World Journal
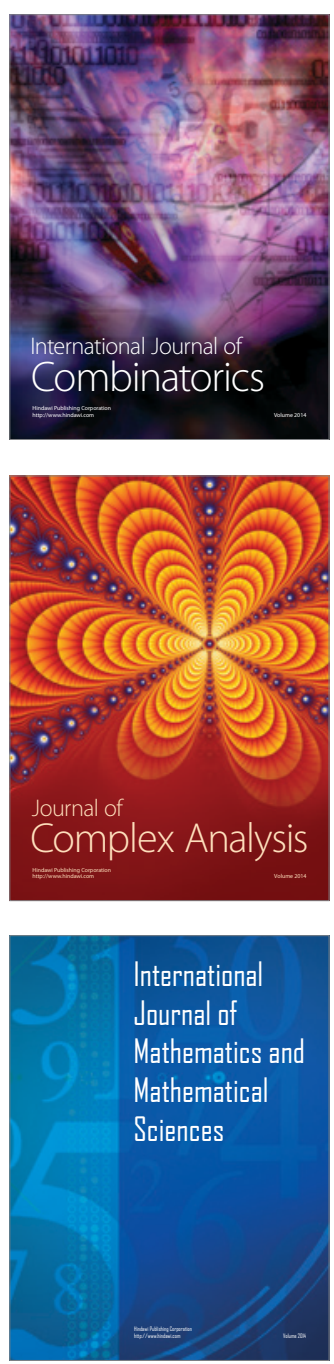
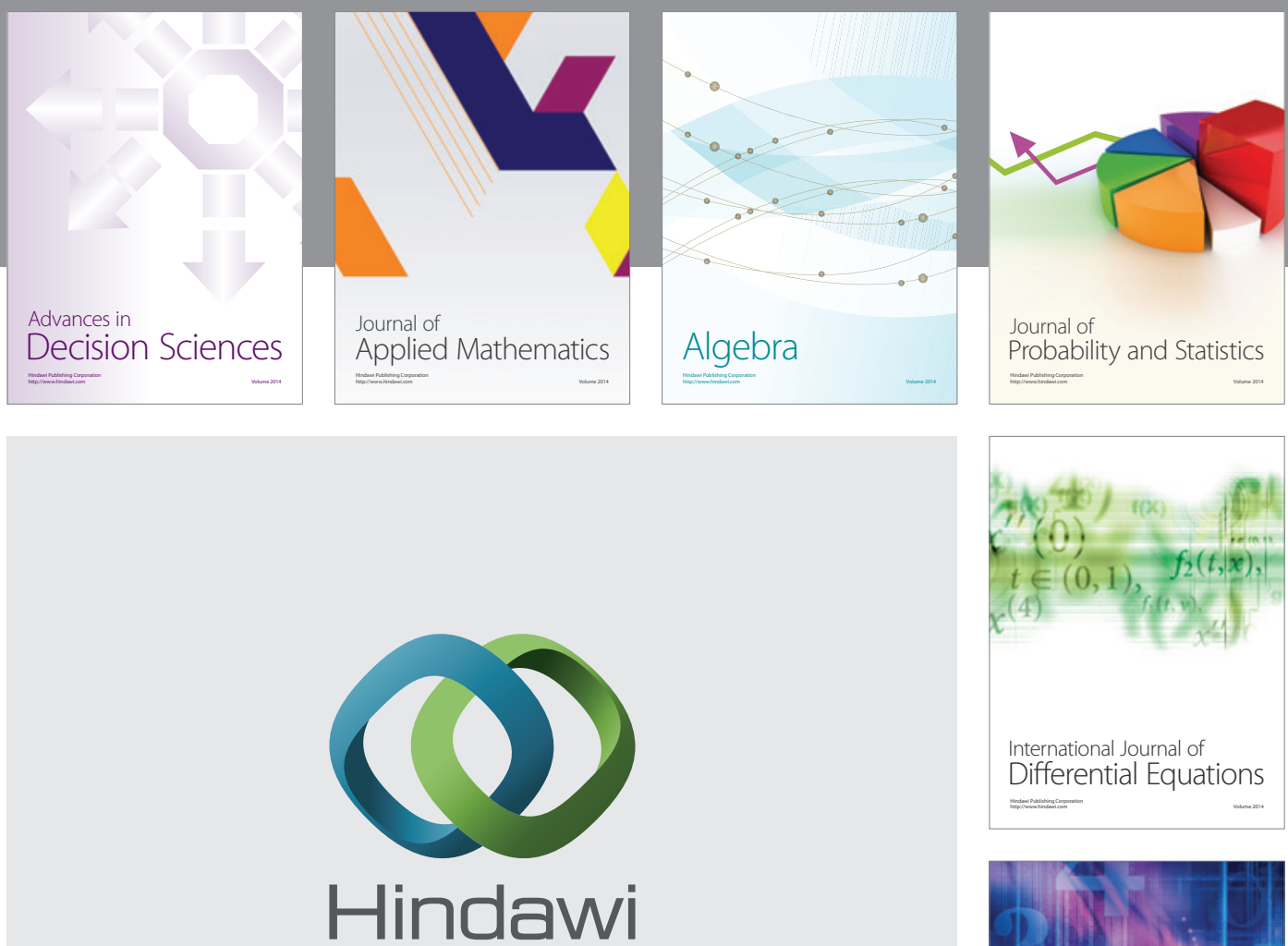

Submit your manuscripts at http://www.hindawi.com
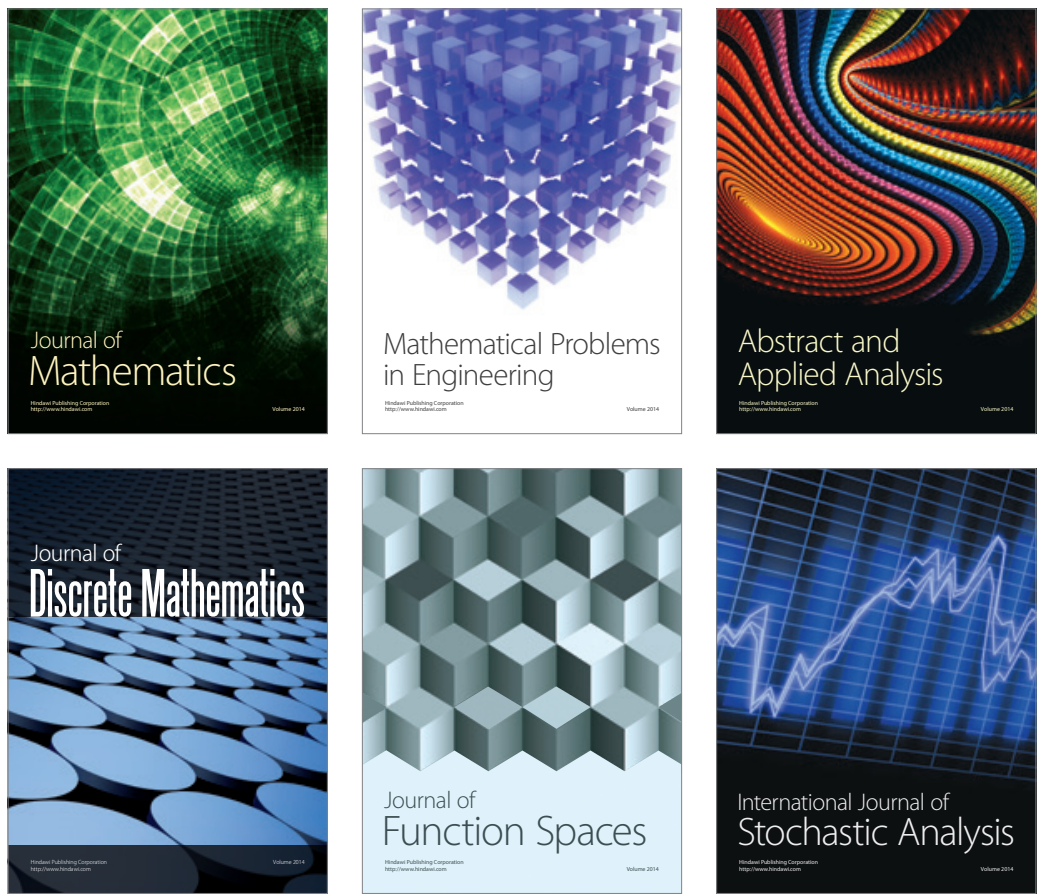

Journal of

Function Spaces

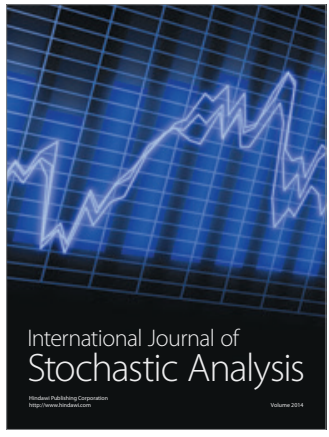

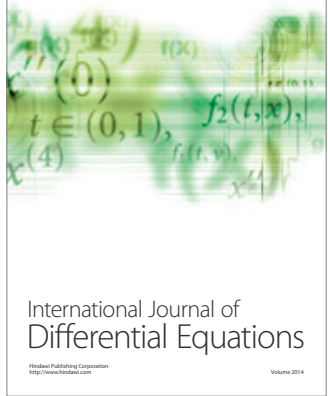
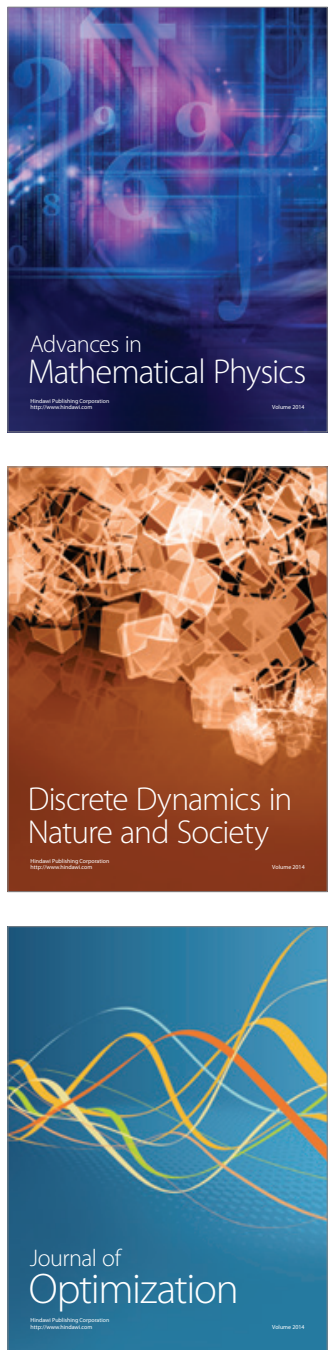